\title{
AGN with faint broad line regions: Some 'True'-Seyfert 2s might be Narrow Line Seyfert 1s
}

\author{
Mónica Valencia-S. ${ }^{1 \dagger}$, G. Busch ${ }^{1}$, S. Smajić ${ }^{1}$, N. Fazeli ${ }^{1}$, \\ S. Behroozian ${ }^{1}$, J. Zuther ${ }^{1}$, S. Fischer ${ }^{2}$ and A. Eckart ${ }^{1,3}$ \\ ${ }^{1}$ I. Physikalisches Institut, University of Cologne, \\ Zülpicher Str. 77, 50937 Cologne, Germany \\ $\dagger$ email: mvalencias@ph1.uni-koeln.de \\ ${ }^{2}$ Deutsches Zentrum für Luft- und Raumfahrt (DLR), \\ Königswinterer Str. 522-524, 53227 Bonn, Germany \\ ${ }^{3}$ Max-Planck-Institut für Radioastronomie, \\ Auf dem Hügel 69, 53121 Bonn, Germany
}

\begin{abstract}
The intriguing existence of 'True'-Seyfert 2s has opened a debate on the validity of the unified model of active galactic nuclei (AGN). These objects, also called 'Naked'-AGN, seem to lack a broad line region. In some cases, their X-ray emission is unabsorbed, typical of Seyfert 1 galaxies, indicating a clear view toward the nucleus, but no broad lines are seen in the optical. In 'True'-Seyfert 2s with higher column densities, observations of polarized light have failed to reveal the hidden broad emission. We performed high-resolution near-infrared integralfield observations of two 'True'-Seyfert 2 candidates: IRAS $01072+4954$ and NGC 7496. We found that the AGN in these sources might power very faint and narrow broad emission lines $\left(F_{W H M}\right.$ broad $\left.<1500 \mathrm{~km} \mathrm{~s}^{-1}\right)$. Here, we discuss the properties of the broad components estimated from published X-ray and/or optical measurements and present their near-infrared candidate identification. Both galaxies host intermediate-mass black holes, with masses $\sim 10^{5}-10^{6} \mathrm{M}_{\odot}$. Our results favor the unified model in the cases of high accretion rates, and stress the necessity of a multiwavelength approach to unveil the nature of 'Naked'-AGN.
\end{abstract}

Keywords. galaxies: Seyfert, galaxies: fundamental parameters, techniques: high angular resolution, line: profiles, galaxies: individual (IRAS 01072 + 4954, NGC 7496)

\section{Introduction}

Active galactic nuclei (AGN) correspond to a phase(or phases) in the evolution of a central massive black hole $(\mathrm{BH})$ and its host galaxy when the central engine is able to excite continuum and line emission all along the electromagnetic spectrum. Although most of the details regarding the AGN physics are still unknown, the unified model seems to provide a good approximation to their geometrical configuration. Nevertheless, AGN are not all the same. Differences are expected e.g., when the central source is in one state or another. AGN without a broad line region (BLR) have been postulated to explain, among other phenomena, the absence of polarized hidden broad lines (HBL) in some Seyfert 2s (e.g. Tran et al. 2010). Some authors suggest that these 'True'-Seyfert 2s, or non-HBL Sy2s, might provide an evolutionary link between starbursts and Seyfert galaxies (Wu et al. 2011). However, the confirmation of the existence of these objects requires a thorough analysis of simultaneous multiwavelength observations, which in general are not available. Some critics to the current evidences are discussed by Antonucci (2012). Polarized broad lines are not expected in optically classified Seyfert 2s that are 
actually Type 1 AGN obscured by dust from the host galaxy, like in the case of NGC 7172 (Smajić et al. 2011). Dust lanes can be difficult to identify even in high quality images, but near-infrared (NIR) spectroscopy can reveal broad emission lines.

In general, observations alone can not be used to prove the absence of broad components, but only to determine upper limits. Challenging the unified model in this respect requires making predictions of the expected broad-line properties and falsify them with the observations. This is possible for 'True'-Seyfert 2 candidates that show multiwavelength characteristics typical of Type 1 objects. In these cases, the null hypothesis is that they are normal Seyfert 1 galaxies, and therefore their AGN properties (like bolometric luminosity, width of the broad lines, BH mass, etc.) follow the same relations as other Type 1 AGN. For example, the bolometric luminosity can be estimated directly from the unobscured hard X-rays, $L_{\mathrm{bol}} \approx 10 \times L(2-10 \mathrm{keV}$ ) (more precise relations in Marconi et al. 2004, Hopkins et al. 2007). From a sample of $~ 3600$ Type 1 AGN from the SDSS, Stern \& Laor (2012) find that the luminosity of the broad $\mathrm{H} \alpha, L\left(\mathrm{H} \alpha_{\text {broad }}\right)$ increases linearly with the X-ray luminosity, $L(2-10 \mathrm{keV}) \approx 130 \times L\left(\mathrm{H} \alpha_{\text {broad }}\right)$, and assuming virialization of the BLR gas, the width and luminosity of the broad components are related to the BH mass as (their Eq. 2) $\log \left(\frac{M_{\mathrm{BH}}}{\mathrm{M}_{\odot}}\right)=7.40+2.06 \log \left[\frac{\mathrm{FWHM}\left(\mathrm{H} \alpha_{\mathrm{broad}}\right)}{1000 \mathrm{~km} \mathrm{~s}^{-1}}\right]+0.545 \log \left[\frac{L\left(\mathrm{H} \alpha_{\mathrm{broad}}\right)}{10^{44} \mathrm{erg} \mathrm{s}^{-1}}\right]$. These relations can be used to predict the expected flux and width of broad-emission lines and other properties of the AGN when the hard X-ray flux and BH mass are known.

\section{2. 'True'-Seyfert 2 candidates}

IRAS $01072+4954$ and NGC 7496 have narrow-line ratios in the composite region of the optical diagnostic diagrams. Although they are not classified as Seyferts, their broad and blue [OIII] line-profiles reveal the presence of the AGN in low-resolution optical data (Moran et al. 1996, Jones et al. 2009). IRAS 01072 is unobscured in the $\mathrm{X}$-rays and displays short and long term variability (Panessa et al. 2005). Emission from hot dust at $T \approx 1200 \mathrm{~K}$ (close to sublimation) confirms the clear view toward the central engine. In this source, the expected properties of the broad lines have been estimated using the expressions presented in Sect. 1 from its hard X-ray luminosity $L(2-10 \mathrm{keV})=10^{41.5} \mathrm{erg} \mathrm{s}^{-1}$, and its $\mathrm{BH}$ mass $M_{\mathrm{BH}} \sim 10^{5} \mathrm{M}_{\odot}$. To scale the properties of the $\mathrm{H} \alpha$ and $\mathrm{Br} \gamma$ broad components, we assumed $L\left(\mathrm{H} \alpha_{\text {broad }}\right) \approx 100 \times L\left(\mathrm{Br} \gamma_{\text {broad }}\right)$, and $\operatorname{FWHM}\left(\mathrm{H} \alpha_{\text {broad }}\right) \approx \operatorname{FWHM}\left(\mathrm{Br} \gamma_{\text {broad }}\right)$. On the other hand, NGC 7496 exhibits an extreme hot dust emission ( $T \sim 1600 \mathrm{~K}$, Prieto et al. 2002) indicative of its Type 1 nature, but only an upper limit of the hard X-ray luminosity $L(2-10 \mathrm{keV})<10^{41.73} \mathrm{erg} \mathrm{s}^{-1}$

Table 1. Flux and width of the broad emission lines in two True-Seyfert 2 candidates: $\operatorname{Br} \gamma$ in IRAS $01072+4954$, and Pa $\alpha$ in NGC 7496. The expected values are derived from their X-ray $[\mathrm{X}]$ or optical $[\mathrm{O}]$ emission. The observed ones are measured from the faint broad-line candidates in the NIR spectra (see Fig. 1 and text for details).

\begin{tabular}{|c|c|c|c|c|}
\hline & \multicolumn{2}{|c|}{ IRAS $01072+4954 \quad(\operatorname{Br} \gamma)$} & \multicolumn{2}{|c|}{ NGC $7496 \quad(\mathrm{~Pa} \alpha)$} \\
\hline & Expected & Observed & Expected & Observed \\
\hline $\begin{array}{l}\text { Broad line flux } \\
\quad\left[\mathrm{erg} \mathrm{s}^{-1} \mathrm{~cm}^{-2}\right]\end{array}$ & $\begin{array}{l}3.2 \times 10^{-16} \\
{[\mathrm{X}]}\end{array}$ & $\begin{array}{l}\sim 1.0 \times 10^{-16} \\
\mathrm{~S} / \mathrm{N} \sim 2.8\end{array}$ & $\begin{array}{l}<7.5 \times 10^{-14} \\
{[\mathrm{X}]}\end{array}$ & $\begin{array}{l}\sim 9.1 \times 10^{-15} \\
\mathrm{~S} / \mathrm{N} \sim 3.7\end{array}$ \\
\hline $\begin{array}{c}\text { Broad line FWHM } \\
{\left[\mathrm{km} \mathrm{s}^{-1}\right]}\end{array}$ & $\begin{array}{l}435-600 \dagger \\
{[\mathrm{X}]}\end{array}$ & $\sim 420$ & {$[\mathrm{O}] \ddagger$} & $\sim 1100$ \\
\hline
\end{tabular}

Notes:

$\dagger$ Two different estimations using relations of Stern \& Laor (2012). The scatter around these values is $\sim 0.3$ dex. $\ddagger$ Derived using the relations of $\mathrm{Kim}$ et al. (2010) from the $\mathrm{FWHM}\left(\mathrm{H} \beta_{\mathrm{broad}}\right)$. 

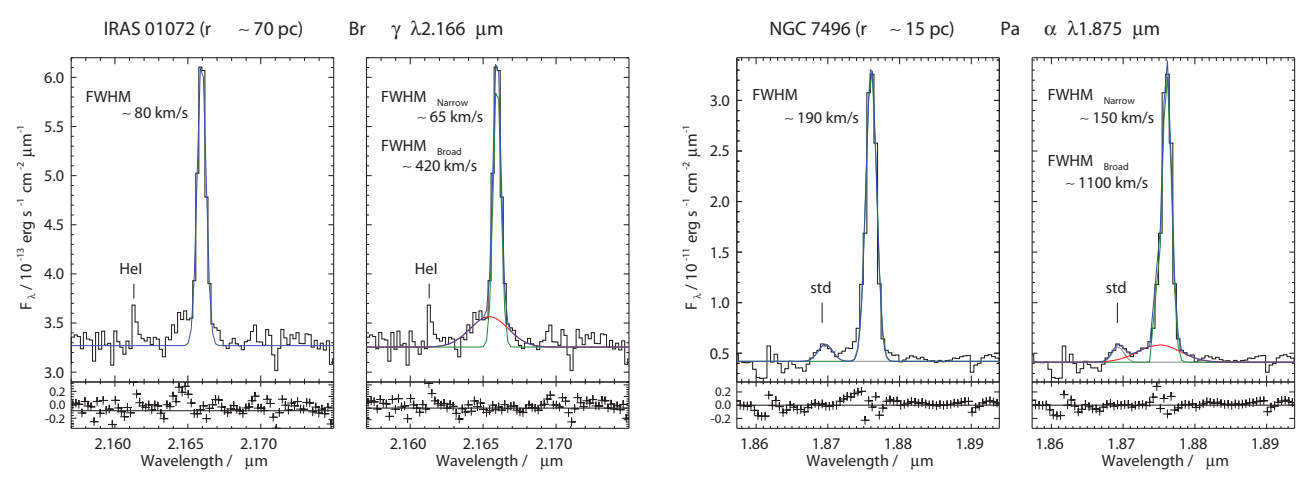

Figure 1. Brightest hydrogen lines in the spectra extracted from apertures with the size of a PSF, centered on the nucleus of both galaxies. Left: $\mathrm{Br} \gamma$ in IRAS $01072+4954$. Right: $\mathrm{Pa} \alpha$ in NGC 7496. Only narrow and narrow+broad Gaussian components and the overall fit are shown. In the bottom panels, the residuals (data - model) are marked with crosses.

is reported in the literature(Polletta et al. 1996). The failed X-ray detection might be caused by a thin dust lane crossing the line of sight, or an intrinsically faint AGN. The optical spectrum of this source reveals faint broad features only after carefully accounting for the stellar continuum contribution (Valencia-S. et al. in prep.). The optical data is not flux calibrated, i.e. the width of the broad components can be determined, but not their luminosity. An upper limit of the expected Pa $\alpha_{\mathrm{broad}}$ broad-line flux can be estimated from the X-rays. We used the relations found by Kim et al. (2010) to estimate the width of the $\mathrm{Pa} \alpha_{\text {broad }}$ from the fitted broad $\mathrm{H} \beta$ emission. Table 1 (cols. 2 and 4) summarizes the expected NIR broad-line properties in both sources, under the assumptions that they are normal Seyfert 1s and the used scale-relations are also valid for low-luminosity AGN.

We performed NIR integral-field observations of the central $\sim 3^{\prime \prime} \times 3^{\prime \prime}$ of both galaxies. IRAS 01072 was observed with NIFS at Gemini North in H- and K-bands at a resolution of $R \sim 5290$. NGC 7496 was observed with SINFONI at ESO-VLT with the $\mathrm{H}+\mathrm{K}$ filter reaching $R \sim 1500$. In both cases, the assistance of laser-guided adaptive-optics systems allowed us to resolve structures down to 0 "' 15 . The brightest hydrogen lines in the NIR spectra are $\mathrm{Br} \gamma$ in IRAS 01072, and Pa $\alpha$ in NGC 7496. The spectral regions around these lines are shown in Fig. 1. A closer look at the base of to the lines reveals 'humps' that we interpret as broad-line candidates. Valencia-S. et al. (2012) disregarded a substantial contribution from other species to this emission in IRAS 01072. However, other interpretations of the observed data are possible. Panels 1 and 2 show the case of single, narrow emission components, while panels 3 and 4 include a broad Gaussian component in the fit. The fact that two components produce a better fit than a single one is expected and does not provide by itself any physical meaning to these 'humps'. However, following the null hypothesis, we considered them as broad-line candidates and measured their width and fluxes to be compared with the expected values derived previously. The measurements are reported in Table 1 (cols. 3 and 5).

\section{On the nature of the sources}

IRAS $01072+4954$ and NGC 7496 host intermediate-mass BHs. The masses were estimated from the $M_{\mathrm{BH}}-\sigma_{*}$ relation (derived by Gültekin et al. 2009) and the stellar velocity dispersion measured in the NIR. In the case of IRAS $01072, \sigma_{*} \approx 35 \mathrm{~km} \mathrm{~s}^{-1}$ gives $M_{\mathrm{BH}} \sim 10^{4.8} \mathrm{M}_{\odot}$ (Valencia-S. et al. 2012). In NGC 7496, $\sigma_{*} \sim 78 \mathrm{~km} \mathrm{~s}^{-1}$ overestimates the $\mathrm{BH}$ mass, $M_{\mathrm{BH}}<10^{6.4} \mathrm{M}_{\odot}$, because the contribution from the rotation of the highly 
inclined galatic disk $\left(\sim 52^{\circ}\right)$ has not been removed. Only for comparison, we calculated the $\mathrm{BH}$ masses from the measured properties of the observed broad-line candidates assuming a virialized BLR (and Eqs. in Sect. 1). The results, $10^{4.7} \mathrm{M}_{\odot}$ for IRAS 01072 and $10^{5.3} \mathrm{M}_{\odot}$ for NGC 7496 agree with those derived from the $M_{\mathrm{BH}}-\sigma_{*}$ correlation. Using the $M_{\mathrm{BH}}-L_{\mathrm{K}}$ relation (Marconi \& Hunt 2003) results in comparatively higher $\mathrm{BH}$ masses, but the validity of this correlation for Type 1 disk galaxies is under debate (see e.g. Busch et al. 2014).

Are the observed 'humps' indeed broad line components? The comparison presented in Table 1 does not prove that the observed extra-emission at the base of the lines originates in the BLR. However, it shows that, if these sources behave like typical Type 1 AGN, the expected broad lines would look very similar (in flux and width) to the 'humps' observed in the data. Are these sources 'True'-Seyfert 2s? The high resolution observations in the NIR do not falsify the null hypothesis, i.e. they look still like normal Seyfert 1s. Are they Narrow Line Seyfert 1 galaxies (NLSy1s)? These two AGN are hosted by blue, barred, disk galaxies. Their BH masses are low and their broad-line widths are narrow $\left(\mathrm{FWHM}_{\text {broad }}<1500 \mathrm{~km} \mathrm{~s}^{-1}\right)$. All these characteristics correspond to those of NLSy1s. However, recently Valencia-S et al. (2013) argued that none of these properties is unique nor ubiquitous of this group of AGN, but are probably related to the low- $M_{\mathrm{BH}}$ and selection biases. We prefer, also in this case, to conclude in favor of the null hypothesis: IRAS 01072 + 4954, as well as NGC 749 and other NLSy1s, are normal Seyfert 1 galaxies.

Acknowledgements. We thank R. Antonucci for interesting discussions on the nature of non-HBLR Sy2s. MV-S acknowledges the support of the DAAD for the participation in the Symposium and the funding from the European Union FP7(2007-2013) under grant agreement No.312789. GB is member of the Bonn-cologne Graduate School and acknowledges support from the Konrad-Adenauer-Stiftung. We thank fruitful discussions with members of the European Union funded COST Actions MP0905 and MP1104. Part of this work was supported by the DFG project number SFB 956.

\section{References}

Antonucci, R. 2012, A\&AT, 27, 557

Busch, G., Zuther, J., Valencia-S., M. et al. 2014, A\&A, 561, A140

Gültekin, K., Richstone, D. O., Gebhardt, K., et al. 2009, ApJ, 698, 198

Hopkins, P. F., Richards, G. T., \& Hernquist, L. 2007, ApJ, 654, 731

Jones, D. H., Read, M. A., Saunders, W., et al. 2009, MNRAS, 399, 683

Kim, D., Im, M., \& Kim, M. 2010, AJ, 724, 386

Marconi, A., Risaliti, G., Gilli, R., et al. 2004, MNRAS, 351, 169

Marconi, A. \& Hunt, L. K. 2003, ApJ (Letters), 589, L21

Moran, E. C., Halpern, J. P., \& Helfand, D. J. 1996, ApJS, 106, 341

Panessa, F., Wolter, A., Pellegrini, S., et al. 2005, ApJ, 631, 707

Polletta, M., Bassani, L., Malaguti, G., et al. 1996, ApJs, 106, 399

Prieto, M. A., Reunanen, J., \& Kotilainen, J. K. 2002, ApJ (Letters), 571, L7

Smajić, S., Fischer, S., Zuther, J., \& Eckart, A. 2012, A\& A, 544, 105

Stern, J. \& Laor, A. 2012, MNRAS, 423, 600

Tran, H. D., Lyke, J. E., \& Mader, J. A. 2011, ApJ (Letters), 726, L21

Valencia-S., M., Zuther, J. \& Eckart, A., et al. 2013, ArXiv: astro-ph/1305.3273

Valencia-S., M., Zuther, J. Eckart, A., et al. 2012, A\&A, 544, 129

Wu, Y.-Z., Zhao, Y.-H., \& Meng, X.-M 2011, ApJS, 195, 17 